\title{
Wireless Telephony.
}

\author{
By Prof. W. H. Eccles.
}

W IRELESS telephony has made such rapid progress during the past six or seven years that it must now be looked upon as a possible rival to wireless telegraphy for communication over distances up to a thousand miles. Although telephonic communication demands on normal occasions the expenditure of more power than does communication by Morse signs, yet the superior rapidity with which thought can be conveyed by voice transmission is a weighty advantage; and, besides, telephony often proves more successful than telegraphy when strays and analogous disturbances are bad, partly because the ear is so skilful in followino the voice in the midst of other noises, and partly because the context greatly assists comprehension. Many of the recent improvements by which the present position in wireless telephony has been reached are due to the development of the thermionic vacuum valve with three electrodes-called, for short, the triode.

The essential difference between wireless telephony and wireless telegraphy is that the voice is used instead of the Morse key to produce alterations in the radiated electric waves. In continuous-wave wireless telegraphy the Morse key, and in wireless telephony the voice, may be applied in two ways: (I) for altering the wavelength, and (2) for altering the amplitude, of the oscillations in the antenna. A distant receiving station capable of sharp response to the normal wave-length of the sending station picks up less energy from the altered waves passing over it, whichever type of alteration is in use at the sending end; for if the amplitude at the sender is altered, the amplitude of the electric and magnetic fields produced at the receiver is changed correspondingly, while if the wave-length at the sender is altered, the receiving station responds less, because the incoming waves are out of tune with it. In many telegraph and telephone systems both types of alteration occur together.

Once the source of continuous waves is available, the main problem in wireless telephony is to provide means of exciting the transmitting antenna in accordance with acoustic vibrations produced by the voice. The process of moulding the oscillatory currents by means of the voice has come to be called "modulation," and the apparatus used, if distinct from the rest of the transmitting apparatus, is called the "modulator."

The obvious method of modulating a given high-frequency alternating current is to use the familiar apparatus of ordinary line telephony. In our ordinary line telephone services direct current is passed through a carbon microphone, and is constant in value so long as the granules in the microphone are quiescent, but when the granules are made to vibrate by the voice, the current is correspondingly modulated. and may be made to produce sound by the familiar telephone receiver consisting of an electromagnet and an iron dia- phragm or reed. In wireless telephony the microphone may be used in a precisely analogous way by being placed in the antenna as shown in Fig. I, or in an earlier circuit as shown in Fig. 2. In the apparatus of these diagrams the oscillatory current may come from an arc, an alternator, or a triode.

A different method of modulating a given source of supply was advocated, especially by R. A. Fessenden in America, early in the history of wireless telephony. The essence of this method was the employment of a condenser of which one surface could be moved relatively to the other by the voice, and this was usually associated with the antenna of the sender. Alterations of the electrical capacity of the condenser produce departures from resonance, and therefore alter the amplitude excited in the antenna by the source of oscillations. On the other hand, the condenser may be used in the circuit generating the oscillations, especially when the source is an arc or a triode, and in this case the frequency of the oscillations supplied to the antenna is modulated by the voice, and conse-

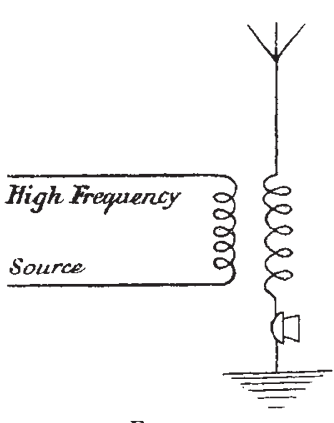

FIG. I.

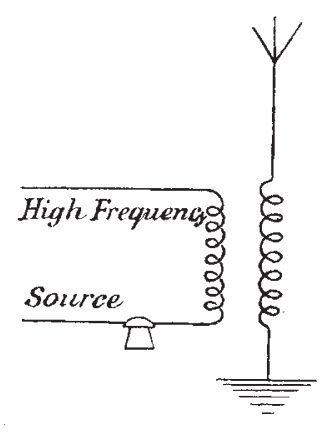

FIG. 2. quently both amplitude and frequency of the oscillations in the antenna are modulated. The condenser has to be of special construction in order that its capacity shall be variable at a frequency of rooo per second. In the recorded experiments it has consisted of a thin diaphragm placed very close to a fixed parallel plate, and the diaphragm has been acted upon either directly by the voice or indirectly by means of some magnified microphone currents passing through an electromagnet.

The above two methods accomplish modulation by variation of the resistance and of the capacity respectively of one or other of the oscillatory circuits. It is natural to consider the possibility of varying the remaining electrical magnitude-namely, the inductance, self or mutual. The variation of self-inductance has been employed by both German and American experimenters, but perhaps the most successful is that due to E. F. W. Alexanderson, of the General Electric Co. of America. In a broad sense this experimenter takes advantage of the dependence of the permeability of iron upon the intensity of the magnetic No. 2643 , VOL. 105] 
field applied to it. Upon the same core of finely laminated iron there is a winding to carry highfrequency current and one for the microphone current. The microphone current as it varies takes the iron to different magnetic states, alters the permeability accordingly, and therefore varies the self-inductance of the high-frequency coils. Many matters of detail have had to be worked out in perfecting the apparatus; an important one

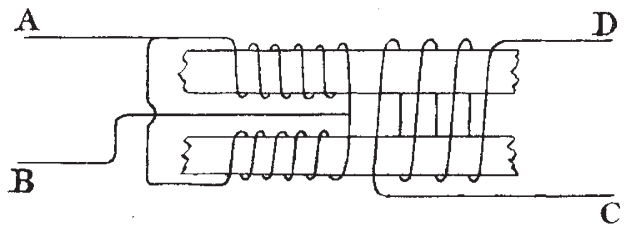

FIG. 3 .

may be explained by aid of Fig. 3. Here A, B are the terminals of the high-frequency circuit, and $\mathrm{C}, \mathrm{D}$ those of the direct-current or microphone circuit. The iron core seen in the figure is part of a closed magnetic circuit; it is in two portions, each of which carries half of the high-frequency winding; the microphone winding encircles both portions of the core. Since the high-frequency windings are wound to exert opposite magnetomotive forces on the halves of the core, they induce negligible high-frequency electromotive forces in the microphone coil.

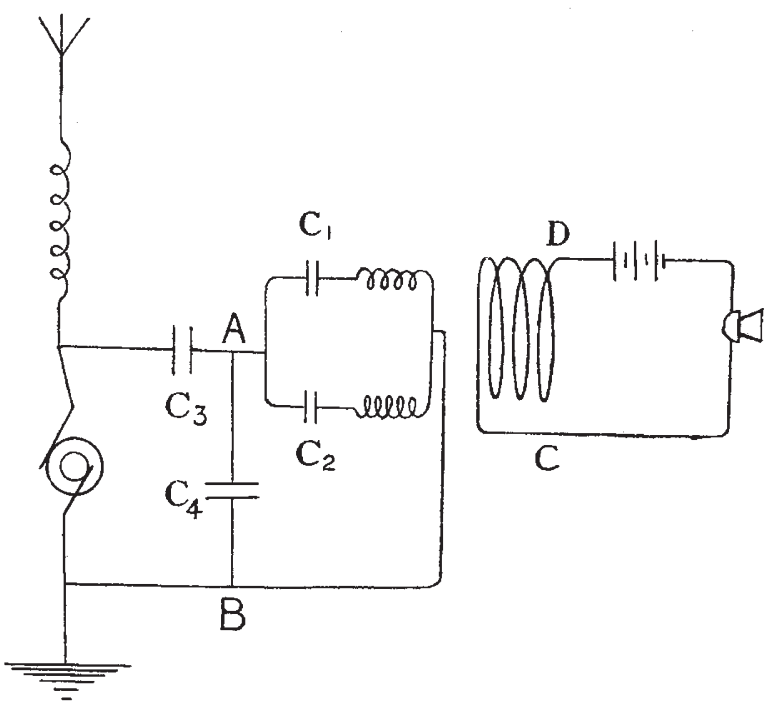

FIG. 4 .

The connections of the apparatus to the alternator, the antenna, and the microphone are shown in Fig. 4, which also introduces further details, namely, the four condensers. For simplicity the windings are represented without their cores, though it must be remembered that the operation of the device depends entirely upon the magnetic properties of NO. 2643 , VOL. IO5] iron. The condensers $\mathrm{C}_{1}$ and $\mathrm{C}_{2}$ have as their chief function the prevention of the flow of undesired acoustic currents in the high-frequency windings. The condenser $\mathrm{C}_{3}$ tends to annul some of the non-varying inductance in the circuit comprising the magnetic controller and the alternatory and the condenser $\mathrm{C}_{4}$ appears to have been introduced for phase adjustment, and enhances the sensitiveness of the whole arrangement to change of microphone current. The performance of the device is excellent; it is stated that a variation of 0.2 ampere in the direct current through the microphone has been made to alter the power given to the antenna from about $6 \mathrm{kw}$. to $43 \mathrm{kw}$.

It was by aid of this device that Ministers in Washington conversed with President Wilson in mid-Atlantic, the voice currents reaching the magnetic controller after passing over land wires from Washington to the New Brunswick wireless station. It will be noticed that in this method of varying an element of the complete antenna circuit the variation is not effected directly by the voice, and in this respect the method is unlike the former two methods.

A related class of methods of modulating the oscillations of the antenna is that in which a voice-varied mutual inductance is employed to transfer the high-frequency energy from the source to the antenna. Perhaps the nearest approach to such a method is that of Kühn, of the Telefunken Co. of Germany, though in his method the self-inductance of the circuits is varied also. The method has not worked out so successfully as that of Alexanderson, and need not be described here.

A third class of methods of modulation aims at varying the activity of the source of highfrequency current; this is in contrast with the preceding methods, in which the functioning of the source is not directly controlled. In the methods to be described the point of application of the control is, so to speak, behind the source, the antenna being supposed to be in front. For example, the direct current that creates the magnetic field of a high-frequency alternator, or the direct current or voltage supplied to one of the circuits of a triode oscillator-that is, an oscillatory circuit sustained in oscillation by means of a three-electrode vacuum valve-might be varied by the voice, and the high-frequency output to the antenna be varied accordingly. Many very miscellaneous schemes have been described; the difficulty is to make a representative selection. The triode oscillator especially lends itself to a multitude of ingenious designs.

In Fig. 5 the circuits of a simple form of oscillator are sketched. The coil marked $\mathrm{L}$ is connected at one end to the anode, at the other to the grid, of a triode, the filament being connected to a tapping in the coil. The inductance $L$ of the coil and the electrical capacity $\mathrm{C}$ of the condenser constitute the circuit in which oscillations are to be maintained. The action of the circuits may be explained broadly thus: Suppose an oscillatory 
current to be flowing in $\mathrm{L}$ and $\mathrm{C}$, and that we choose an instant when the grid is, say, at a positive potential relative to the filament and rising in value; in accordance with the properties of these tubes the electromotive force transferred from the grid to the anode circuit of the tube will be from filament to anode outside the tube and rising in value. Thus the electromotive force acting on the portion of the coil in the anode circuit is in phase with the potential difference postulated to exist throughout the coil in virtue of the oscillating current in it, and therefore the electrical motion tends to be maintained.

In such an oscillator the frequency of the oscillations is mainly determined by the inductance and capacity, but every other circumstance of the circuits has its influence. Moreover, the amplitude of the oscillations often varies when the frequency does. Thus if acoustic variations be imposed upon the current employed for heating the filament. or upon the electromotive force in the anode circuit, or upon a source of electromotive force between grid and filament, the highfrequency output of the assemblage varies correspondingly. An ordinary microphone will, it need scarcely be said, be used for converting the voice-made air vibrations into current varia-

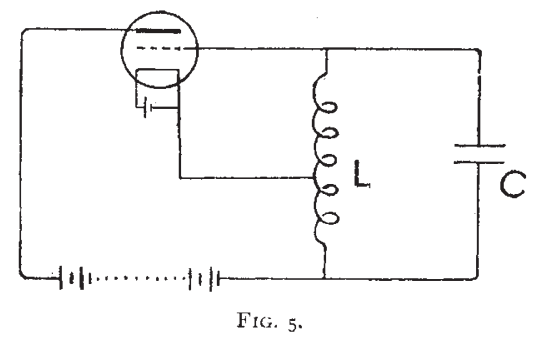

tions, and transformers will be used when of advantage for introducing the current variations into the various circuits mentioned above.

One of the most interesting of these methods was described by Major Prince in a paper read recently before the Wireless Section of the Institution of Electrical Engineers, the apparatus being that finally adopted by the R.A.F. for aeroplane work.

The principles employed may be seen from Fig. 6, which is drawn as free from detail as possible. The antenna $A$ is maintained in oscillation by the transmitting triode $R_{1}$ in a manner similar to that of Fig. 5 ; the modulator tube $R_{2}$ magnifies the voltages applied to its grid by the microphone currents in the primary of the transformer $T$. The high voltage for the anode circuits of each tube is supplied by the battery $\mathrm{E}$, which is in series with a large choking coil $\chi$. When the microphone is quiescent the full voltage of $\mathrm{E}$ acts steadily on the anode of $R_{1}$, but when the microphone produces variable electromotive forces on the grid of $R_{2}$ these are multiplied, transferred to the anode circuit of $\mathrm{R}_{2}$, and if the choking coil is large enough to be effective, and the condenser $C$ not too large, they reach the anode circuit of $R_{1}$. In consequence the amplitude of the oscillaNO. 2643 , VOL. IO5] tions generated by $R_{1}$ is varied in correspondence with the microphone current variations, and to a small extent the frequency is changed also. This set of apparatus is styled a 20 -watt set.

When the normal range of transmission must attain roo miles, the problem of modulating the necessary power becomes formidable, chiefly on account of the limitations affecting the microphone. It is obvious that direct modulation by means of ordinary microphones is impossible except for small currents, say not exceeding 2 amperes; in consequence, in the endeavour to achieve long-range telephony, special microphones - some of them employing liquid conductorshave been devised, and sometimes many microphones have been used in parallel. At this stage the three-electrode vacuum valve comes to our assistance in various ways, some of which must now be explained. In the first place the triode may be employed as an amplifier of the variable currents or electromotive forces leaving the microphone, and these may be applied to the

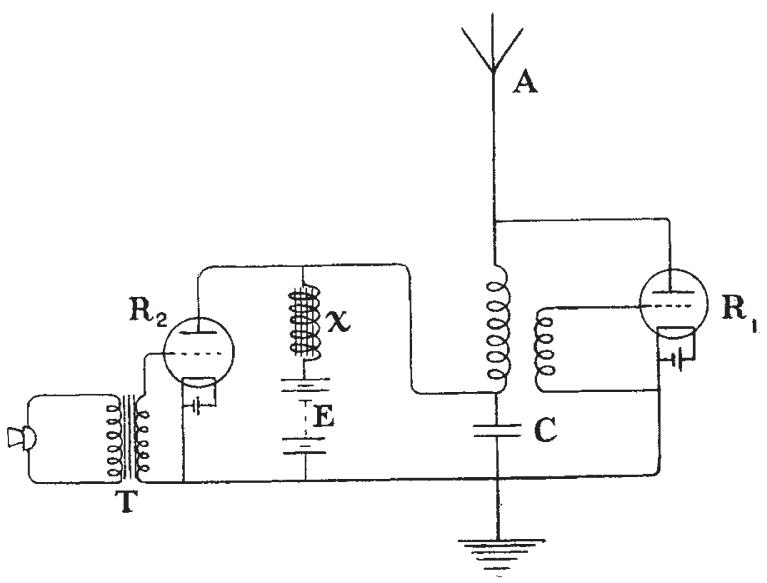

FIG. 6.

modulation of larger high-frequency currents than was before possible; the tube is then the actual modulator. An instance of this appears in Fig. 6. In the second place the triode may be utilised as a by-pass to deflect high-frequency current from the antenna to itself or to other apparatus capable of dissipating the necessary amount of energy-a subtraction method that has proved very successful. The tube may serve in either or both of these functions as modulator of the high-frequency current from arcs, alternators, or other sources of oscillations, not omitting the triode oscillator itself. Evidently the fact that these three-electrode valves can perform the distinct offices of generator of oscillations, magnifier of high and low frequencies, and modulator, and in each office may be associated with a remarkable variety of circuits, may be expected to lead, and is leading, to endless permutations and combinations in the circuits proposed for medium- and long-range wireless telephony.

There is space for brief descriptions of only two examples of large power plants. In r9r4 
the Western Electric Co. conducted a great experiment from the American naval station at Arlington, near Washington, and succeeded in speaking to the Eiffel Tower (3700 km.), and exceptionally to. Honolulu $(8000 \mathrm{~km}$.). Triodes were used as oscillators, modulators, and magnifiers. The chain of apparatus was as follows: First came a triode oscillator of small power, which was coupled by means of a highfrequency transformer to the grid circuit of a medium-sized tube. This grid circuit contained also the secondary winding of a transformer, the primary of which carried the currents from the microphone. The anode circuit of this medium triode therefore contained magnified modulated current of the high frequency dictated by

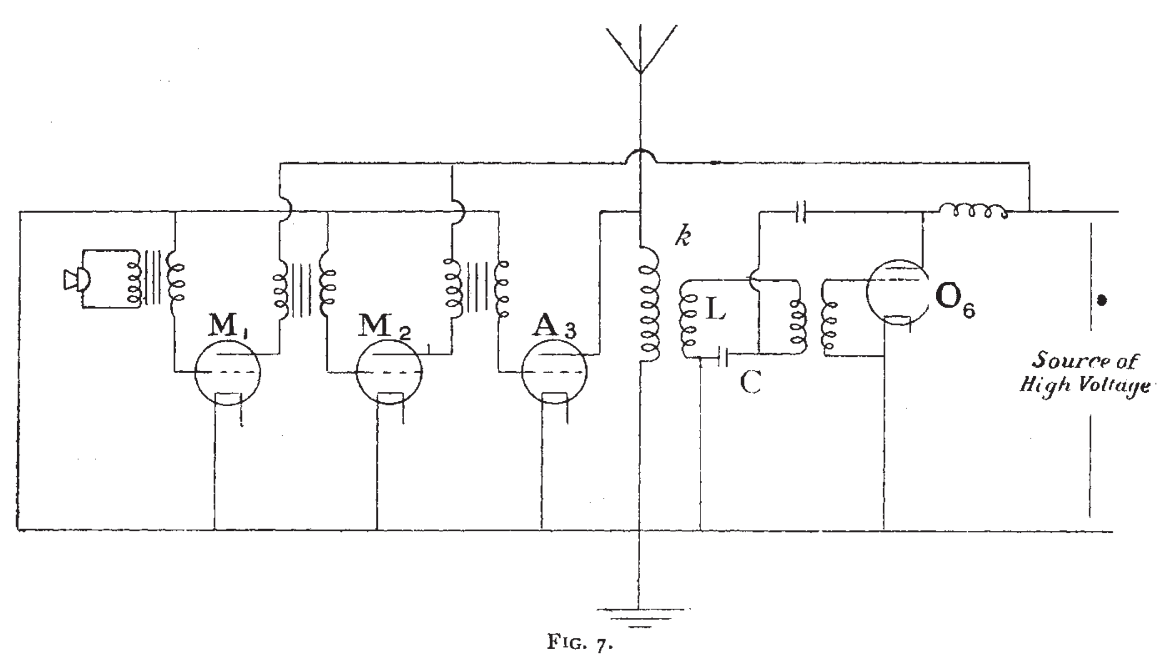

type. A diagram of the chief parts appears in Fig. 7, from which are omitted all details regarding the heating currents for the filaments and concerning the rectufying of the high-voltage current for the anode circuits. The oscillations are generated in the circuit $\mathrm{LC}$ by a bank of six large three-electrode valves in parallel marked $\mathrm{O}_{0}$, and transferred to the antenna by the coupling $k$. The absorption tubes are three in number, and are shown at $\mathrm{A}_{3}$; their three anodes are large enough to dissipate all the energy normally given to the antenna. These absorption triodes are controlled by applying to their grids the speech electromotive forces after these have been magnified by the successive tricdes $M_{1}$ and $M_{2}$. The total consumption of power is $20 \mathrm{kw}$., including all that necessary for heating the filaments, the height of the aerial is $400 \mathrm{ft}$., and the wavelength $275^{\circ}$ metres.

It will be noticed that the above are all oneway methods-that is to say, the two persons using two stations for conversation must speak in turn, and the listener must wait for the speaker to finish before he switches over from his listening circuit to his speaking circuit. This falls far short of perfection. For perfect telephony it is essential that both persons shall

the small triode oscillator. The current was next transferred by means of a transformer from this anode circuit to the grid circuit of a bank of medium-sized amplifying tubes connected in parallel, and was again magnified, and finally it was transformed once more into a circuit containing the grids of more than 500 parallel connected tubes. It ought to be remarked that great progress has been made since 1914 in the manufacture of power bulbs, and that the experiment can now be carried out on a larger power scale with a far smaller array of tubes.

The remaining example to be cited is the recently erected $20-\mathrm{kw}$. plant of the Marconi Co. During the past few weeks it has transmitted good quality speech to a distance of 1500 miles. In principle it is of the subtraction or absorption be able to speak and hear simultaneously if they so desire, as in ordinary line telephony. For information about the most modern attempts at duplex telephony a paper read recently before the Institution of Electrical Engineers by Mr. P. P. Eckersley should be consulted. Until a complete duplex method is devised and proved, wireless telephony must remain a somewhat irksome mode of communication for public use. The ideal method will be such that a wireless station on this side of the Atlantic could be connected over land lines to, say, London, and a station on the other side connected by land lines to New York, and the persons using the apparatus would be unable to tell that wireless telephony across the ocean had been an agency in the transmission of their voices.

\section{The Meteorology of the Temperate Zone and the General Atmospheric Circulation.}

\section{By Prof. V. Bjerknes.}

IN Norway, since the year I9I8, an attempt has been made to base forecasts of weather on the application of a very close network of meteorological stations. The study of the corresponding very detailed synoptic charts has led to interesting results even for large-scale meteorNO. 2643 , VOL. IO5] ology. These are due especially to three young meteorologists, J. Bjerknes, H. Solberg, and T. Bergeron, who have been attached to this service, and will return to the subject in detailed papers.

A very short summary of some of the main 